\title{
Sprouts Emerging from the Dendrites of Axotomized Lamprey Central Neurons Have Axonlike Ultrastructure
}

\author{
Garth F. Hall, Ann Poulos, and Melvin J. Cohen \\ Department of Biology, Yale University, New Haven, Connecticut 06511
}

We have examined the dendritic and axonal ultrastructure of intact anterior bulbar reticulospinal neurons (ABCs) in the CNS of the larval sea lamprey and compared it with that of the dendrites and neuritic sprouts from ABCs examined 2 months following axotomy. Dendrites and axons of intact $A B C$ s are distinguishable from one another by several ultrastructural criteria: (1) the predominance of microtubules in the dendritic cytoskeleton and neurofilaments in that of the axon, (2) the exclusively postsynaptic status of the dendrites versus the presynaptic status of the axon, and (3) the presence of polyribosomes and large numbers of mitochondria in the dendrites and their respective absence and scarcity in the axon.

The ultrastructure of axonal sprouts evoked by axotomy of $A B C s$ 1-1.5 mm from their somata ("intermediate axotomy") in many ways resembled that of intact axons. Axonal sprouts were presynaptic to other neurons, and their cytoskeletons consisted mainly of neurofilaments. They also exhibited some features not seen in either axons or dendrites, such as numerous clusters of small vesicles that were not associated with synapses and, in some cases, close associations with glial elements. We also examined sprouts emerging from the dendrites of $A B C s$ following axotomy within $\mathbf{5 0 0} \mu \mathrm{m}$ of their somata ("close axotomy") and found that such "dendritic" sprouts closely resembled axonal sprouts; they possessed neurofilament-dominated cytoskeletons, were presynaptic to other neurons, and were often associated with glial elements.

The dendrites of $\mathrm{ABC}$ s undergoing dendritic sprouting retained their normal gross morphology but possessed a mixture of "axonal" and "dendritic" ultrastructural characteristics, exhibiting neurofilament-dominated cytoskeletons while remaining entirely postsynaptic to other neurons. However, there were significantly fewer synapses on the dendrites of axotomized cells than were found on the dendrites of intact ABCs.

We conclude that sprouts evoked by axotomy are intrinsically axonal in character whether they originate from the axon stump or from the dendritic tree. Our results also suggest that the materials necessary for axonal regeneration may displace elements of the dendritic cytoskeleton as they are transported through the dendrites to the emerging "dendritic" sprouts following close axotomy.

\footnotetext{
Received Mar. 9, 1988; revised June 23, 1988; accepted June 27, 1988.

This work was supported by NIH Spinal Trauma Grant 2P50 NS 10174 and NIH Physiology Training Grant GM 15727

Correspondence should be addressed to Garth F. Hall, Department of Zoology, University of California, Berkeley, CA 94720

Copyright $(1989$ Society for Neuroscience $0270-6474 / 89 / 020588-12 \$ 02.00 / 0$
}

The division of neuronal processes into 2 different classesdendrites and axons - is of fundamental importance to the function of most neurons. The distinctive properties of dendrites and axons are responsible for the polarization of signal traffic in neurons and are thus probably essential for the generation of normal behavioral patterns by groups of neurons. Axons and dendrites of vertebrate neurons differ characteristically from one another in features of their gross morphology, ultrastructure, and immunocytochemistry (Peters et al., 1976; Matus et al., 1981; Bartlett and Banker, 1984), making it possible to study the development and maintenence of neuronal polarity on several different levels of analysis.

Differentiation of axons and dendrites may be controlled by both intrinsic and environmental factors. It has been shown that cultured hippocampal neurons can develop well-defined axons and dendrites in the absence of external cues (Bartlett and Banker, 1984). On the other hand, the identity of processes in this system can also be respecified by external influences such as axotomy (Dotti and Banker, 1987). Chamak et al. (1987) have shown that the nature of the glial substrate can also play a role in determining whether cultured neurons develop axons or dendrites.

In this study, we have used axotomy as a way of challenging a neuron to replace its missing axon both with the correct type of process (i.e., an axon) and at the correct location within the cell. The extent to which a neuron is able to do this under various experimental circumstances should shed light on the problem of how a neuron creates and maintains the characteristic form and specializations of its neuritic processes.

Axotomy usually causes the injured cell to stop producing many of the materials used in normal neuronal function and to bcgin synthesizing materials necessary for the regeneration of a new axon (Grafstein and MacQuarrie, 1978). In most cases, these materials are directed to the correct site for axonal regeneration; i.e., the cut end of the proximal axon stump. Recently, however, a number of instances in both invertebrates and vertebrates have been found in which axotomy has resulted in sprouting from other parts of the cell, notably the dendrites (Hall and Cohen, 1983, 1988a; Roederer and Cohen, 1983; Linda et al., 1985), the stumps of cut dendrites (Hall and Cohen, 1988b) and the soma (Murphy and Kater, 1980; Schacher and Proshansky, 1983). In all of these cases, the gross morphology and trajectories of the sprouting neurites resembled those of axons rather than dendrites. These observations have raised the issue of whether axotomy always stimulates the production of specifically "axonal" materials regardless of where sprouts originate within the cell or, alternatively, if sprouts emerging from the dendrites have "dendritic" properties that are masked by environmental factors. 
There are a number of characteristic ultrastructural differences between the dendrites and axons of most vertebrate neurons that can be used to distinguish intrinsically axonlike from dendritelike processes. Dendrites usually contain many of the same organelles found in the cell body, including those involved in protein synthesis, such as rough endoplasmic reticulum (rER) and free polyribosomes. These organelles are absent from the axons of almost all neurons, thus providing one fairly reliable criterion for distinguishing the 2 types of processes (Peters et al., 1976; Bartlett and Banker, 1984). The cytoskeletons of dendrites and axons are also typically different from one another; those of dendrites are usually composed mainly of microtubules while those of axons (particularly large axons) contain many more neurofilaments (Lasek et al., 1983). Finally, dendrites and axons can usually be distinguished on the basis of their synaptic relations with other cells; dendrites are usually postsynaptic to other neurons, while axons form presynaptic elements.

In this study, we have examined the ultrastructure of neuritic sprouts originating from both the axon stump and dendritic tips of axotomized ABCs. We used the criteria listed above to assess whether the sprouts originating from dendrites possess an "axonal" or "dendritic" identity. Our conclusion is that the ultrastructure of sprouts emerging from dendrites strongly resembles that of both axonal sprouts and intact axons, while differing greatly from the dendrites of intact cells. This suggests that "dendritic sprouting" is indeed the result of the rerouting of materials synthesized for axonal regeneration into the dendrites rather than either the stimulation of new dendritic growth or the production of sprouts without an intrinsically defined identity.

\section{Materials and Methods}

All experiments were performed on large (9-12 cm) larval sea lampreys (Petromyzon marinus) collected from stream beds in the Connecticut River basin. Animals were maintained in aerated aquaria at $4{ }^{\circ} \mathrm{C}$ and were provided with several inches of alluvial substrate for burrowing. Axotomy of anterior bulbar cells (ABCs) was performed as described by Hall and Cohen (1988a). Lampreys were anesthetized by immersion for several minutes in a $0.13 \%$ aqueous benzocaine solution and then transferred to lamprey saline (Wickelgren, 1977) for either "close" (within $500 \mu \mathrm{m}$ of the soma) or "intermediate" (1-1.5 mm from the soma) axotomy (Fig. 1, $C, D$ ). Postoperative lampreys were kept in lamprey saline for $1-2 \mathrm{~d}$ before being returned to well water, where they were kept at $15^{\circ} \mathrm{C}$ until the morphology of the $\mathrm{ABCs}$ was examined with $\mathrm{HRP}$ histochemistry.

Intracellular staining with IIRP. We used intracellular staining with HRP to examine the gross morphology and ultrastructure of intact $\mathrm{ABCs}$ ( 9 cells in 7 animals) and in ABCs subjected to "close" axotomy 57$70 \mathrm{~d}$ previously ( 6 cells in 5 animals) or "intermediate" axotomy $50 \mathrm{~d}$ previously ( 2 cells in 2 animals). These survival periods were chosen to maximize the amount of sprouting occurring from axotomized $\mathrm{ABCs}$ at the time of sacrifice and to minimize the likelihood of dendritic retraction (Hall and Cohen, 1988a). Lampreys were anesthetized as described above, and the brain and $3 \mathrm{~cm}$ of spinal cord were removed and placed in a Sylgard-lined petri dish filled with lamprey saline at 15$18^{\circ} \mathrm{C}$. ABC somata were then impaled under visual guidance with microelectrodes having resistances of 80-150 megohms. Microelectrode tips were filled with $3 \%$ HRP in $0.05 \mathrm{~mm}$ Tris buffer $(\mathrm{pH} \mathrm{7.3)}$ and backfilled with $0.1 \mathrm{M} \mathrm{KCl}$. HRP was injected with $5 \mathrm{nA}, 200 \mathrm{msec}$ positive square-wave pulses at $2.5 \mathrm{~Hz}$ over a period of $6 \mathrm{~min}$. This produced a level of staining that was sufficiently distinct to permit positive identification of HRP-filled processes with both light and electron microscopy, but did not obscure ultrastructural detail. Lamprey brains were maintained for $1 \mathrm{hr}$ in oxygenated saline $\left(4^{\circ} \mathrm{C}\right)$ and then fixed by immersion in $3.5 \%$ glutaraldehyde in $0.1 \mathrm{M}$ sodium cacodylate buffer with $2 \%$ sucrose for $2 \mathrm{hr}$ at $4^{\circ} \mathrm{C}$. Brains were processed for the HRP reaction in the presence of diaminobenzidine according to the protocol of Wood and Cohen (1979). They were then hemisected and viewed under a dissecting microscope. Only brains containing single, well-filled $A B C s$ were selected for electron microscopy. When brains containing axotomized $\mathrm{ABCs}$ were viewed (Fig. $1, C, D$ ), drawings indicating the location of visible sprouts were made. In the case of $A B C s$ subjected to close axotomy (Fig. 1C), brains containing filled cells that possessed axon stumps and/or periaxonal sprouts (Hall and Cohen, 1983) were rejected at this stage in order to avoid later confusion of dendritic sprouts with axonal sprouts.

Electron microscopy. Lamprey brains to be processed for electron microscopy were postfixed in $1 \% \mathrm{OsO}_{4}$ in $0.1 \mathrm{~m}$ sodium cacodylate buffer for $1 \mathrm{hr}$, washed in distilled water, dehydrated in an ethanol series, and then cmbedded in Spurrs medium. Thick $(3 \mu \mathrm{m})$ and thin $(60 \mathrm{~nm})$ transverse sections of the brain were cut from rostral to caudal on a Sorvall MT-2 ultramicrotome. Thick sections were examined under low magnification in the light microscope and were used to identify appropriate regions of the filled cell for subsequent thin sectioning (indicated in Fig. 1). Thin sections for electron microscopy were stained with uranyl acetate and lead citrate and then examined and photographed on a Zeiss 10CA transmission electron microscope operating at $80 \mathrm{kV}$.

Criteria for the identification of sprouts. Dendritic sprouts were readily distinguished from dendrites by their linear, rostrocaudal trajectories and sparse branching, as well as by their locations outside of the normal dendritic limit of ABCs (Hall and Cohen, 1988a). Sprout tips were defined for the purposes of this study either as the region of the sprout within $20 \mu \mathrm{m}$ of its distal end (in the case of sprouts lacking swollen tips) or the swollen region itself (Fig. $1 C$, brackets). Sprout shafts were defined as the region between $50 \mu \mathrm{m}$ of the sprout tip and $100 \mu \mathrm{m}$ of the rostralmost dendrite (Fig. $1 C$, dots). Axonal sprouts from $\mathrm{ABCs}$ subjected to intermediate axotomy were identified by following the axon stump caudally until additional HRP profiles appeared (Fig. $1 D$, dots).

Quantification of ultrastructural features. Measurements of the relative frequency of occurrence of microtubules, neurofilaments, synapses, and mitochondria were madc from micrographs of each section that we examined in the EM. The relative abundance of microtubules and neurofilaments in a given section was determined by placing a ruler perpendicularly across process profiles at randomly chosen sites, counting the number of microtubules or neurofilaments crossed by the ruler edge, and dividing by the total length (in microns) measured by the ruler in the process profile (minus the number of microns covered by mitochondria). An estimate of the relative number of mitochondria present was given by the percentage of the process profile crossing the ruler edge that was covered by mitochondria. Synapse frequency was determined by measuring the total length of the cross section of plasma membrane seen in each micrograph (in microns) and dividing this into the number of synapses counted. The statistical significance of differences between means (Table 1) was calculated on a per cell basis using a nonparametric statistical test - the randomization test for independent samples (Siegel, 1956).

\section{Results}

Normal morphology of $A B C$ processes

The dendrites and axons of intact $\mathrm{ABCs}$ differ systematically from one another in several prominent features of their overall form. The gross morphology of both have been described previously in detail (Fishman, 1975; Martin, 1979; Hall and Cohen, 1983, 1988a). ABC dendrites are highly branched, project ventrally or laterally from the soma along curvilinear trajectories and taper from base to tip. By contrast, the axon is unbranched, projects caudally from the soma along a linear trajectory and cxpands as it reaches the spinal cord, where it maintains a constant diameter. While the dendrites almost always terminate within $350 \mu \mathrm{m}$ of the soma, the axon extends the full length of the spinal cord, a distance of $10 \mathrm{~cm}$ or more in many animals.

We found that the ultrastructure of dendrites also differed greatly from that of the axon in intact ABCs. The dendritic cytoskeleton consisted mainly of evenly spaced microtubules running along the length of the dendrite. Neurofilaments were also present, but were relatively uncommon (Fig. 2, Table 1). 


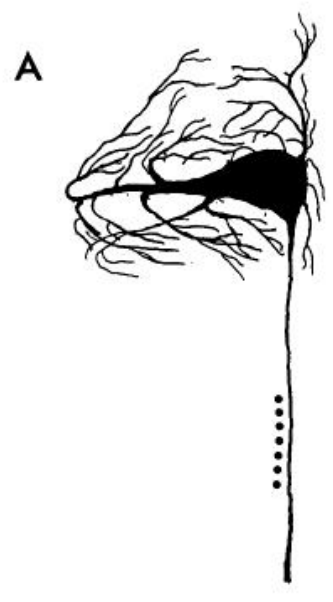

C

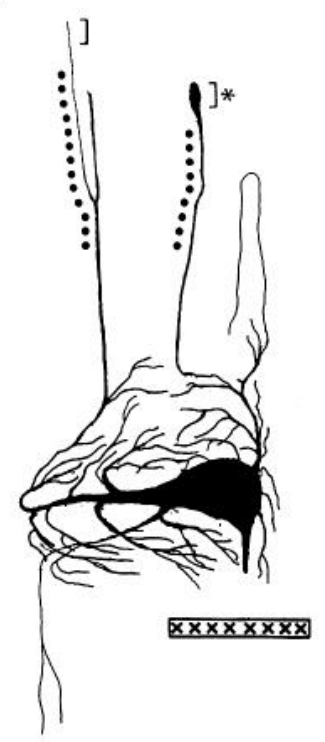

B

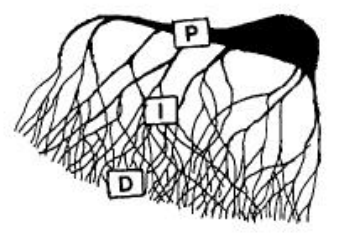

D

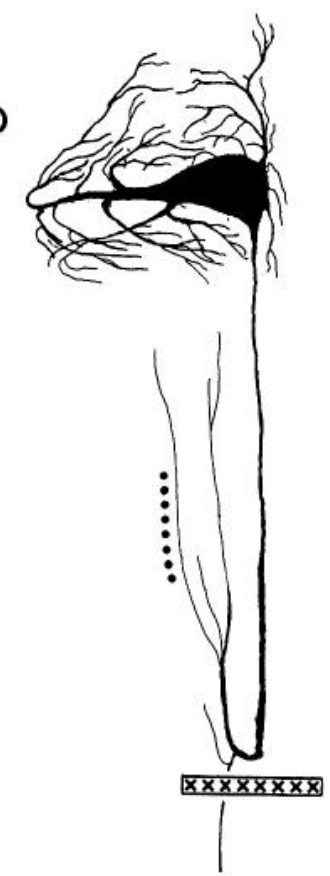

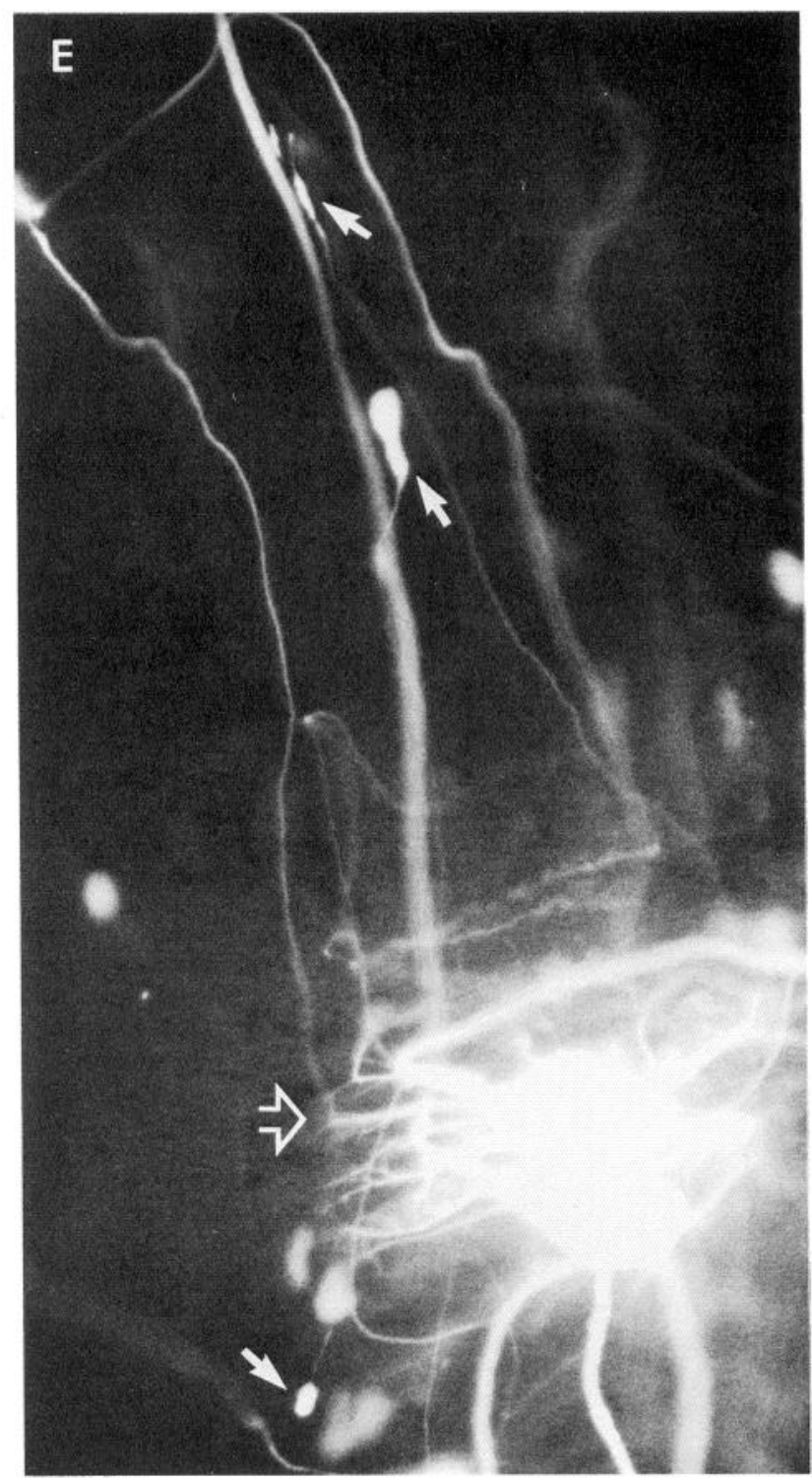

Figure 1. $A-D$, Schematic diagrams of $\mathrm{ABCs}$ showing the locations sampled for transmission electron microscopy of $\mathrm{ABC}$ processes. $B$, Transverse view of the $\mathrm{ABC}$ dendritic tree; $A, C$, and $D$, dorsal views of intact $(A)$ and axotomized $(C, D) \mathrm{ABCs}$. For intact $\mathrm{ABCs}$, sections of axon were taken in the caudal hindbrain $(A$, dots $)$. $B$ indicates location of dendritic sections taken from proximal "P," intermediate "I," and distal " $D$ " regions of the dendritic tree. For ABCs subjected to close axotomy within $500 \mu \mathrm{m}$ of the soma 57-70 d previously $(C)$, sections of dendritic sprouts were taken at distances of $100 \mu \mathrm{m}$ or more from the rostral limit of the dendritic field $(C$, dots $)$. Brackets denote sprout tips, which were classified as either tapered or swollen (asterisk). Sections of axonal sprouts were obtained from ABCs subjected to axotomy $1-1.5 \mathrm{~mm}$ from the soma $50 \mathrm{~d}$ previously $(D, d o t s)$. Sections of dendrite were taken from ABCs subjected to close axotomy 57-70 d previously at the sites indicated in $B$. $E$, Dorsal view of 2 ABCs injected with Lucifer yellow (Stewart, 1978) $105 \mathrm{~d}$ after close axotomy. Rostral is toward the top of the photo. Rostrally projecting sprouts extend well beyond the limits of the dendritic tree. Open arrow, a sprout at its point of origin in the dendritic tree; solid arrows, swollen tips of dendritic sprouts.

Another feature typical of $\mathrm{ABC}$ dendrites was the presence of large numbers of mitochondria (Table 1), particularly near the dendritic tips. Smooth endoplasmic reticulum and free polyribosomes were also visible in some dendrites that had been lightly filled with HRP reaction product (not shown). Synaptic vesicles were not observed in the dendrites of intact ABCs. Axonal ultrastructure differed consistently from that of dendrites in each of these features (Fig. 3A). The axonal cytoskeleton was primarily composed of longitudinally oriented neurofilaments rather than microtubules, mitochondria were less common than in dendrites, and free polyribosomes were not seen. Isolated vesicles of various sizes and smooth endoplasmic reticulum were often visible in the axoplasm, although clusters of small vesicles were only seen in association with chemical synapses. Thus, the ultrastructure of both the dendrites and axons of intact ABCs is similar to that seen in many large vertebrate central neurons (Peters et al., 1976).

$\mathrm{ABC}$ dendrites were invariably postsynaptic to other neurons. We found large numbers of chemical synapses, particularly on the higher-order dendrites, but no evidence of either presynaptic profiles or electrical synapses. Synapses onto ABC dendrites were characterized by slightly convex postsynaptic surfaces (Fig. $2 B$ ), postsynaptic densities of $15-20 \mathrm{~nm}$, and presynaptic densities that were usually somewhat narrower. Synaptic clefts typ- 

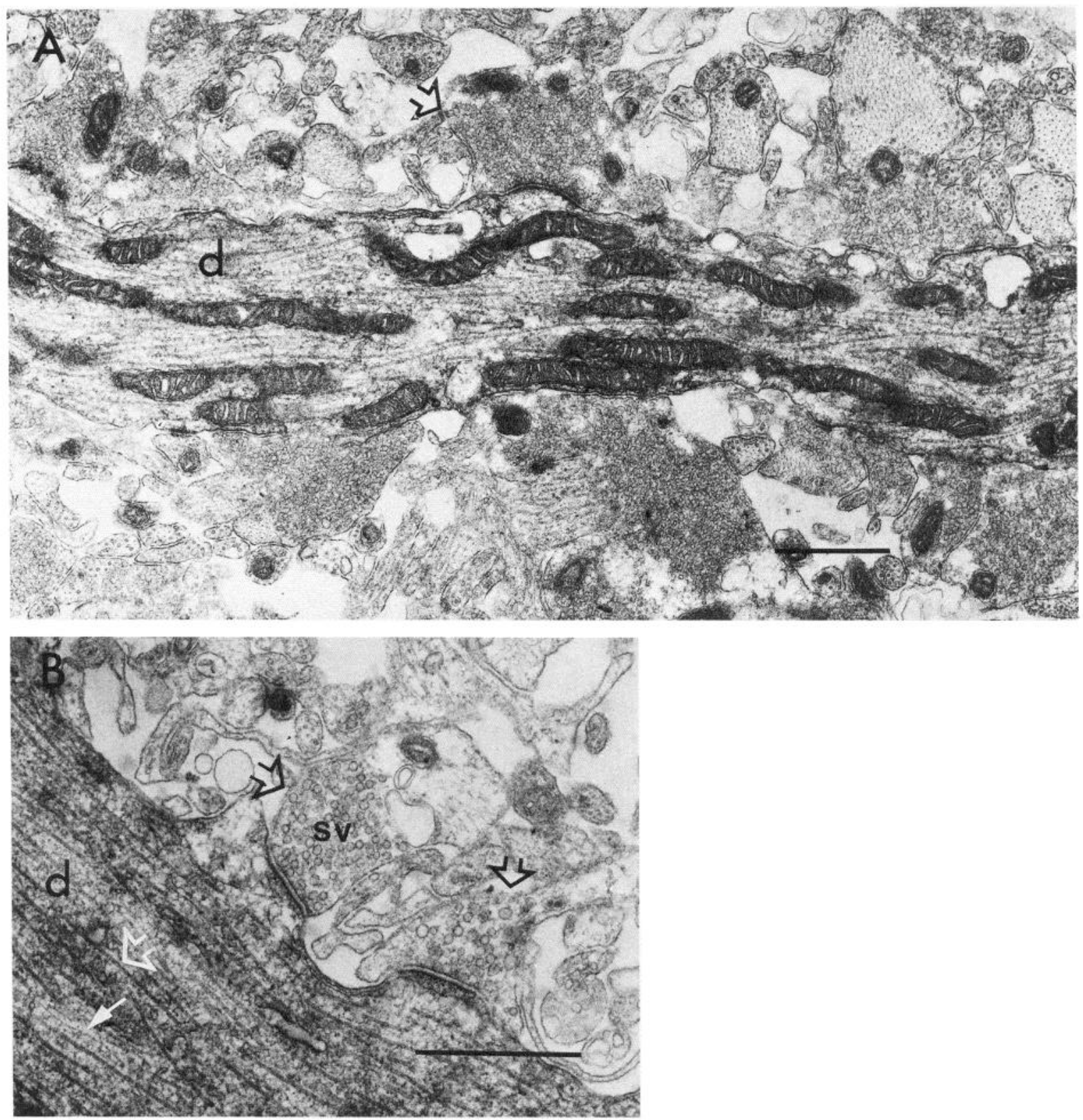

Figure 2. Normal dendritic ultrastructure of an intact ABC filled intracellularly with HRP. A dendrite (d) labeled with HRP is shown in both $A$ and $B$. The dendritic cytoskeleton consists primarily of microtubules $(B$, open white arrow) and has relatively few neurofilaments $(B$, solid white arrow). Mitochondria are common. ABC dendrites are invariably postsynaptic to other cells. Open black arrows, presynaptic terminals; sv, synaptic vesicles. Scale bars, $1 \mu \mathrm{m}$.

ically measured $15 \mathrm{~nm}$ across. The synaptic terminals contained electron-lucent vesicles, which were usually associated with triangular dense projections extending from the presynaptic density. Muller cell axons (including those of ABCs) make en passant synapses on neural elements along their length in the spinal cord (Rovainen, 1974). We found that chemical synapses in normal $\mathrm{ABC}$ axons were always presynaptic to other neural elements (the opposite of the situation in the dendrites) and were sometimes adjacent to electrical synapses. All of the synapses that we observed on normal axons were of the en passant variety and exhibited a raised presynaptic "platform" rather than a presynaptic bouton, with a slight concavity at the site of the synapse (Fig. $3 B$ ).

\section{Morphology of axonal sprouts}

The gross morphology of axonal sprouts following axotomy of $\mathrm{ABCs}$ in the hindbrain is similar in many respects to that of the normal axon (Hall and Cohen, 1988a). These sprouts tend to be linear, rostrocaudally oriented, and cylindrical in outline rather than tapered. Although axonal sprouts do exhibit some branching, they are usually much less branched than are dendrites.

We examined the ultrastructure of 5 sprouts (12 sections) 
Table 1. Ultrastructure of intact and axotomized ABCs

\begin{tabular}{|c|c|c|c|c|c|c|c|c|}
\hline & \multirow[b]{2}{*}{$\begin{array}{l}\text { Cells } \\
(n)\end{array}$} & \multirow[b]{2}{*}{$\begin{array}{l}\text { Sections } \\
(n)\end{array}$} & \multicolumn{2}{|c|}{ Cytoskeleton } & \multicolumn{3}{|l|}{ Synapses } & \multirow{2}{*}{$\begin{array}{l}\text { Mito- } \\
\text { chondria } \\
\text { (\% of } \\
\text { profile) }\end{array}$} \\
\hline & & & $\begin{array}{l}\text { Tubules } \\
\text { (per } \mu \mathrm{m})\end{array}$ & $\begin{array}{l}\text { Filaments } \\
(\text { per } \mu \mathrm{m})\end{array}$ & Polarity & Total & $\begin{array}{l}\text { Frequency } \\
\text { (per } 10 \mu \mathrm{m})\end{array}$ & \\
\hline Intact axon & 6 & 13 & 0.6 & 14.1 & Presynaptic & 8 & 0.4 & 1.8 \\
\hline Intact dendrite & 3 & 18 & 10.9 & 4.7 & Postsynaptic & 113 & 2.0 & 20.4 \\
\hline Dendrite of axotomized cell & 6 & 27 & 0.7 & 21.7 & Postsynaptic & 59 & 0.6 & 6.1 \\
\hline Axonal sproul & 2 & 12 & 0.8 & 24.0 & Presynaplic & 2 & 0.03 & 3.1 \\
\hline \multicolumn{9}{|l|}{ Dendritic sprouts } \\
\hline Total & 6 & 23 & 0.2 & 22.7 & Presynaptic & 6 & 0.075 & 2.0 \\
\hline Shaft & & 14 & 0.2 & 22.7 & & 0 & 0 & 2.3 \\
\hline Tip & & 9 & 0.1 & 22.8 & & 6 & 0.15 & 1.7 \\
\hline
\end{tabular}

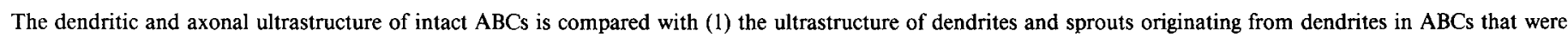

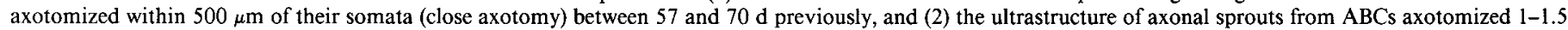

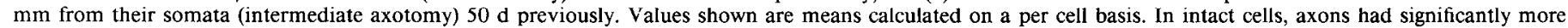

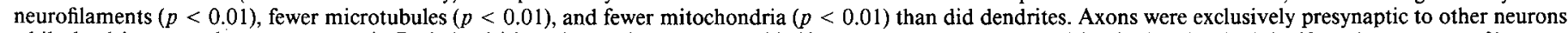

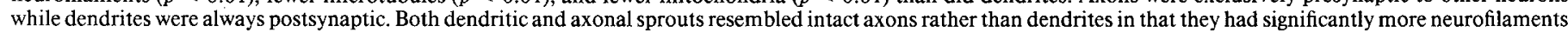

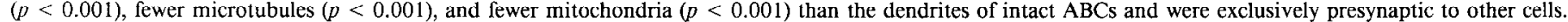

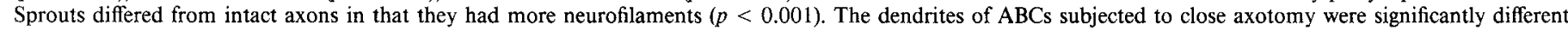

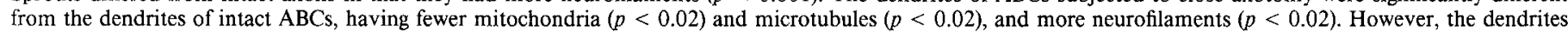

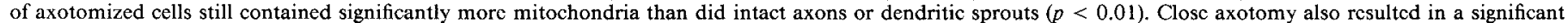
reduction in the number of synapses seen on the dendrites of axotomized cells $(p<0.02)$.

emerging from the axon stumps of 2 ABCs axotomized $50 \mathrm{~d}$ previously at a point $1 \mathrm{~mm}$ away from their somata in the caudal hindbrain. In general, axonal sprouts resembled intact axons in most of their ultrastructural features, as well as in their gross morphology. Sprout cytoskeletons consisted mostly of neurofilaments with very few microtubules, although neurofilaments were more closely packed in sprouts than in intact axons (Fig. $4 A$, Table 1). Mitochondria were relatively scarce, and polyribosomes were not seen. We observed 2 regenerated synapses; these also closely resembled synapses made by intact axons (Fig. $4 B$ ). One feature of sprout ultrastructure that was not present in intact axons was the occurrence of large clusters of vesicles in the cytoplasm of some sprouts (Fig. 4A). The individual vesicles in these clusters closely resemble the vesicles normally associated with chemical synapses in the axon, being round, electron-lucent, and approximately the same size as vesicles found at axonal synapses. However, the vesicle clusters seen in sprouts were often quite large, were not associated with other synaptic features, and were often situated at a distance from the plasma membrane. Some sprouts also appeared to be wrapped in cellular elements resembling the endfeet of radial glia observed in "disorganized" tissue near the lesion site in transected lamprey spinal cords (Wood and Cohen, 1981). This was not seen in intact $A B C$ axons, which are unmyelinated and do not maintain large areas of contact with individual glial elements. On the whole, however, these results indicate that axotomy stimulates $\mathrm{ABCs}$ to replace the missing axon with neurites that exhibit both the gross morphology (Hall and Cohen, 1988a) and ultrastructural specializations peculiar to axons.

\section{Morphology of dendritic sprouts}

It has been shown (Hall and Cohen, 1983, 1988a) that if ABCs are subjected to axotomy within $500 \mu \mathrm{m}$ of their somata ("close axotomy"), most of the resulting sprouts emerge from the dendritic tips rather than from the axon stump (Fig. $1 E$ ). The gross morphology and trajectories of these sprouts are very similar to those of sprouts originating from the axon stump, with both types of sprout being predominately linear and rostrocaudally oriented. Some dendritic sprouts also exhibited large swollen tips 20 or more $\mu \mathrm{m}$ in diameter. We found that the ultrastructure of dendritic sprouts closely resembled that of axonal sprouts. We selected for analysis a total of 23 thin sections of 10 dendritic sprouts ( 6 cells), including both sprout shafts and tips ( 5 of which were swollen). As in both axonal sprouts and intact axons, the cytoskeleton of dendritic sprouts was predominantly composed of neurofilaments, mitochondria were relatively uncommon, and polyribosomes were absent. Dendritic sprouts were similar to axonal sprouts (and unlike intact axons) in that they often contained large clusters of small electron-lucent vesicles not associated with synapses. Both types of sprout also had more densely packed neurofilaments (Table 1) than did intact axons. However, unlike the axonal sprouts that we examined, we only found vesicle clusters in the tips (both swollen and tapered) of dendritic sprouts, usually bordering the plasma membrane. Sprout tips were completely filled with neurofilaments, which often exhibited a swirled appearance quite unlike that of the linear, longitudinally oriented neurofilaments usually seen in sprout shafts. This was particularly true of swollen sprout tips (Fig. $5 B$ ). The glial processes that we noted wrapping some axonal sprouts were also prominently associated with many dendritic sprouts (Fig. 5A).

We observed a total of 6 chemical synapses made by dendritic sprouts. In each case, the sprout was the presynaptic element, providing a striking point of similarity between the ultrastructure of dendritic sprouts and that of both axonal sprouts and intact axons. Furthermore, these synapses formed by sprouts from dendrites (Fig. $5 B$ ) resembled the overall form of normal axonal synapses as seen in Figure $3 B$. They were generally the same length (1-2 $\mu \mathrm{m})$ and exhibited a slightly raised plateau where they made contact with the postsynaptic element, as described for synapses in both normal and regenerating axons (Christensen, 1976; Wood and Cohen, 1981). However, synapses were only seen at the tips of dendritic sprouts (Table 1), a distribution that is quite different from that of synapses along the length of the the intact axon (Batueva and Shapovalov, 1974; Rovainen, 1974) and along regenerating axonal sprouts in the spinal cord (Wood and Cohen, 1981). 


\section{Dendritic morphology of ABCs subjected to close axotomy}

The strong similarity of dendritic sprouts to $\mathrm{ABC}$ axons at both the gross morphological and ultrastructural levels suggests that dendritic sprouting is caused by the intracellular rerouting into the dendrites of materials otherwise destined for axonal regeneration at the axon stump. The growth of axonlike sprouts from the dendritic tips can only occur if specialized "axonal" materials (such as neurofilaments and presynaptic structures) are transported through the dendrites and incorporated into emerging from the dendritic tips. We were therefore curious to see whether dendritic ultrastucture changes following close axotomy, especially as the gross morphology of the dendrites themselves is not visibly affected by this procedure (Hall and Cohen, 1988a).

We found that close axotomy resulted in radical changes in the dendritic ultrastructure of 6 cells (27 sections) examined between 57 and $70 \mathrm{~d}$ postaxotomy. The most prominent result of close axotomy was that the primarily microtubular cytoskeleton seen in normal dendrites was replaced by one composed mainly of neurofilaments in all of the dendrites that we examined. This changeover was due both to a 16 -fold decrease in the density of microtubules and a 4-fold increase in the density of neurofilaments (Fig. 6, Table 1). Neurofilaments were also more densely packed than those of intact axons. The dendritic cytoskeleton thus resembled that of axonal and dendritic sprouts by 2 months or so following close axotomy.

Other aspects of dendritic ultrastructure showed less change following close axotomy. Typically "dendritic" organelles such as polyribosomes were still present in the dendrites of axotomized cells. Mitochondria, however, were significantly fewer than in normal dendrites (Table 1), although they still occurred more frequently than in either axons or sprouts. Most importantly, the dendrites remained entirely postsynaptic to other cells. However, the frequency of synapses onto the dendrites was reduced following close axotomy when compared with normal dendrites (Table 1). The glial elements seen wrapping many dendritic (and axonal) sprouts were not seen on the dendrites themselves, suggesting that there may be a specific interaction of glial cells with actively growing processes following injury.

The "proximal" and "intermediate" dendrites of ABCs subjected to close axotomy always appeared as described above, as did some dendrites that we classified as "distal" dendrites (Fig. $1 B$ ). However, some processes that could be classified as distal dendrites by their diameters, locations, trajectories, and certain ultrastructural features also contained structures similar to those found in axonal and dendritic sprouts (Fig. 6B). These "hybrid" processes exhibited exclusively "dendritic" features (incoming chemical synapses) next to sproutlike features such as vesicle clusters and glial wrappings, suggesting that these processes may be dendritic tips that are just beginning to sprout.

\section{Discussion}

The findings presented in this paper strongly suggest that both dendritic and axonal sprouts evoked by axotomy are intrinsically "axonal" in nature. This is based on the observations that (1) the gross morphology and ultrastructure of normal dendrites and axons are consistently very different, and (2) axonal and

Figure 3. Normal axonal ultrastructure of an intact $\mathrm{ABC}$ following intracellular injection with HRP. $A$, The cytoskeleton consists mainly
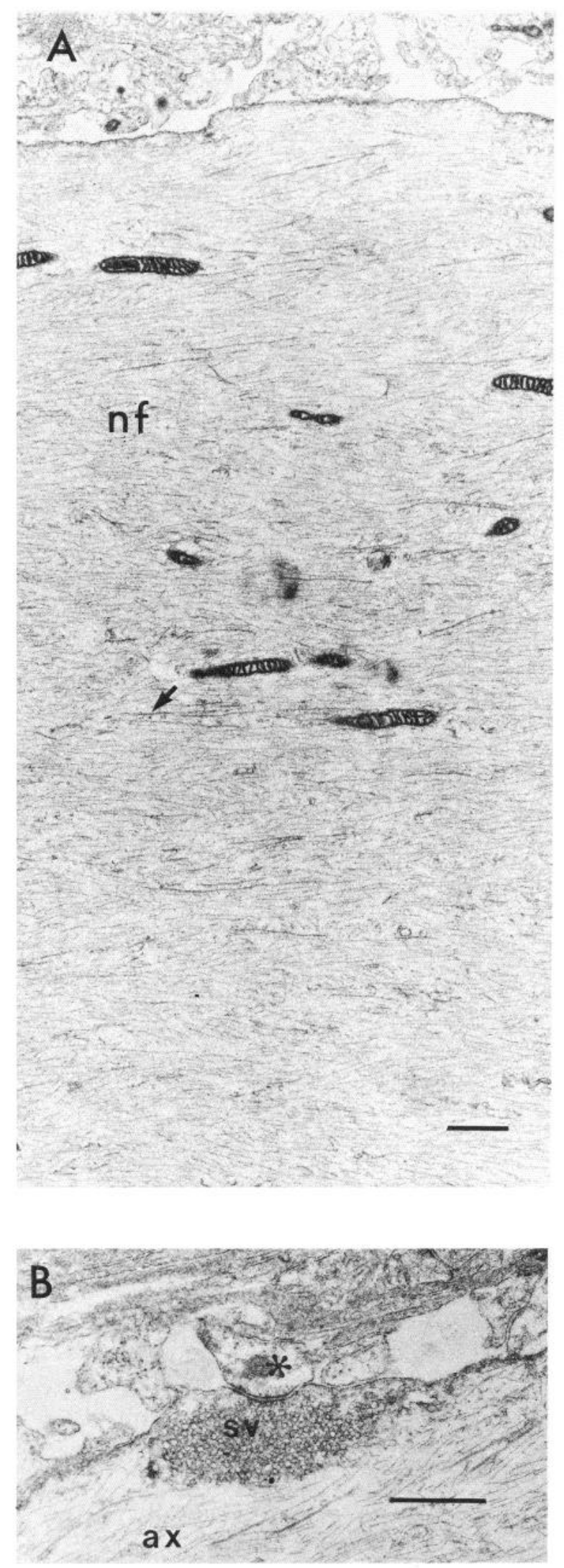

of neurofilaments $(n f)$ with relatively few microtubules (solid arrow) distributed throughout the axon. Mitochondria are scarce compared with dendrites. $B, E n$ passant synapse filled with synaptic vesicles (sv) made by an $\mathrm{ABC}$ axon $(a x)$ onto an unidentified postsynaptic element in the caudal hindbrain. Scale bars, $1 \mu \mathrm{m}$. 

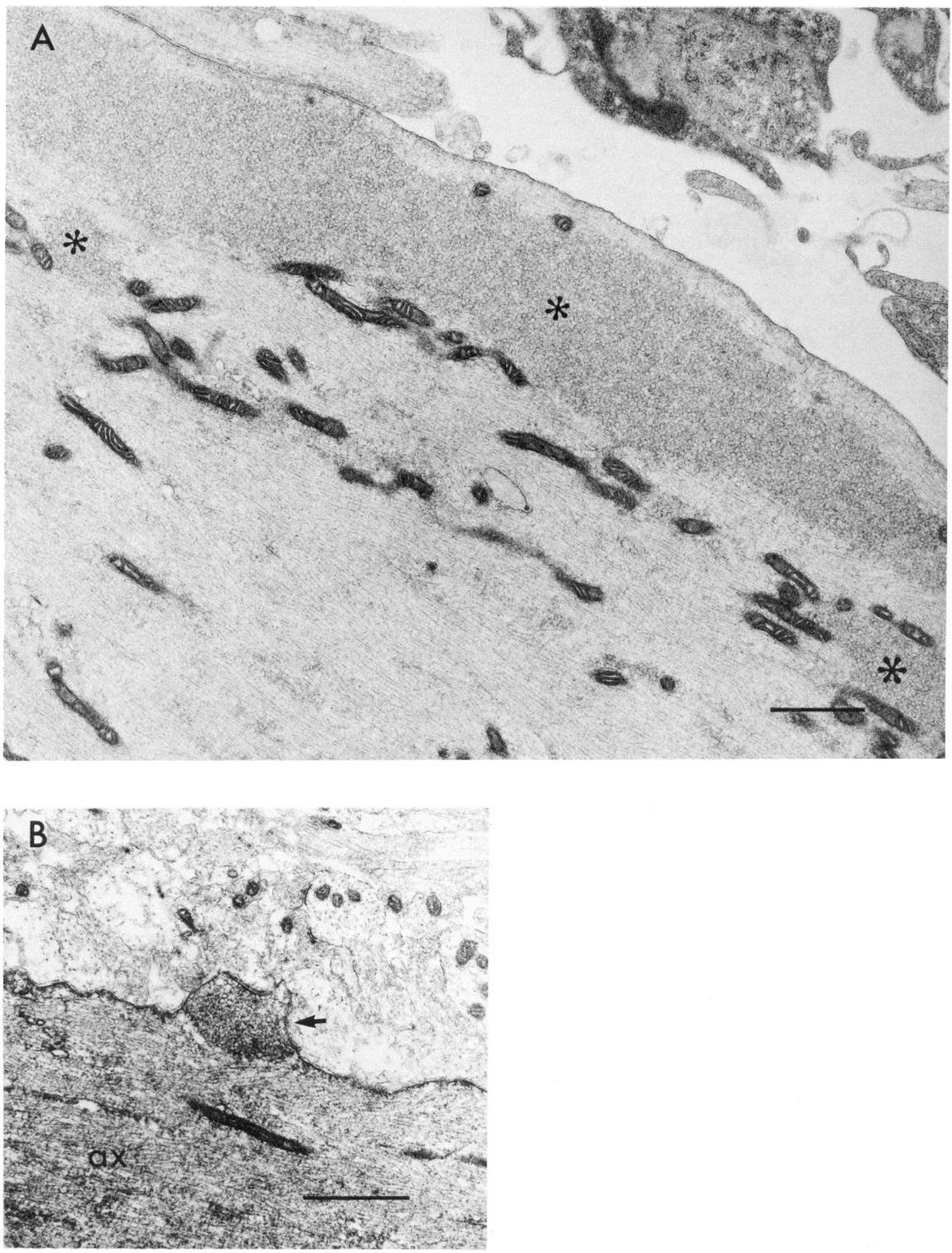

Figure 4. Ultrastructure of HRP-filled axonal sprouts from an ABC axotomized in the caudal hindbrain $50 \mathrm{~d}$ previously. A, Longitudinal section of sprout showing cytoskeleton consisting mainly of neurofilaments. Large groups of vesicles (asterisks) that are not associated with synapses are also present. $B$, Another axonal sprout $(a x)$ from the same cell as in $A$ with a regenerated synapse showing the typical morphology of an ABC axonal synapse. Scale bars, $1 \mu \mathrm{m}$. 
dendritic sprouts are similar to each other and to normal axons in their gross morphology and ultrastructure, and are unlike normal dendrites. Axotomy close to the soma therefore appears to cause material destined for regenerating axonal sprouts to be routed into the dendrites, where it replaces the normal dendritic structure with an axonlike cytoskeleton; this occurs without affecting overall dendritic form or changing the polarity of dendritic synaptic connections.

\section{Characteristics of dendritic and axonal sprouts}

Although dendritic and axonal sprouts evoked by axotomy resemble the intact axon in the most prominent aspects of their gross morphology and ultrastructure, we also observed a number of differences between intact axons and sprouts. Three common features of both dendritic and axonal sprouts that were unlike intact axons were (1) large clusters of vesicles that were not associated with synapses present in some sprouts, (2) more densely packed neurofilaments in sprouts than were present in intact axons, and (3) glial processes that partially wrapped many sprouts.

One possible reason for the abundance of vesicle clusters in sprouts is that they may be the source of membrane needed for sprout elongation. Sprout growth via vesicle exocytosis at the growth cone would require the transport of a large number of vesicles from the soma along the sprout to its tip. Various types of membranous vesicles are a common cytoplasmic component in both developing axons (Chang and Reese, 1986) and in growth cones of neurites in culture (Landis, 1983), where it has been suggested that vesicle exocytosis at the growth cone is a major mechanism of membrane addition (Bray, 1973; Pfenninger and Maylie-Pfenninger, 1981; Pfenninger and Johnson, 1983). It should be noted that the vesicles present in $\mathrm{ABC}$ sprouts resemble synaptic vesicles rather than the larger and more irregular vesicles associated with growth cones in other systems (Del Cerro and Snider, 1968; Bunge, 1973; Skoff and Hamburger, 1974; Pfenninger and Maylie-Pfenninger, 1981; Chang and Reese, 1986). However, there are important similarities between the exocytosis of vesicles at growth cones and at synaptic terminals (Llinás, 1979), both of which appear to be mediated by increased $\mathrm{Ca}^{2+}$ fluxes across the plasma membrane. An increased $\mathrm{Ca}^{2+}$ conductance has been demonstrated in the tips of regenerating Muller axons in the lamprey (MacVicar and Llinás, 1985), suggesting that $\mathrm{Ca}^{2+}$-mediated exocytosis of vesicles is a plausible mechanism of sprout elongation in axotomized ABCs.

Neurofilaments were consistently packed more closely together in both dendritic and axonal sprouts and in the dendrites of ABCs following close axotomy than they were in intact axons. This suggests that subtle changes in either the type of neurofilament proteins present or their assembly may occur following axotomy. These changes may be similar to the developmentally regulated differences seen in the binding of neurofilament-associated proteins (Ciment et al., 1986). For example, the spacing between neurofilaments is thought to be controlled by the length of the cross-bridges between neurofilaments (Lasek et al., 1983).

Figure 5. A, Longitudinal section of a dendritic sprout shaft (spr) taken at a site $150 \mu \mathrm{m}$ from the sprout tip. Note the neurofilament $(n f)$ dominated cytoskeleton and the presence of cellular elements wrapping the sprout (open arrows). Occasional microtubules (solid arrow) are also present. $B$, Section through a swollen dendritic sprout tip (st) showing a synapse in which the sprout contains synaptic vesicles $(s v)$ and is the
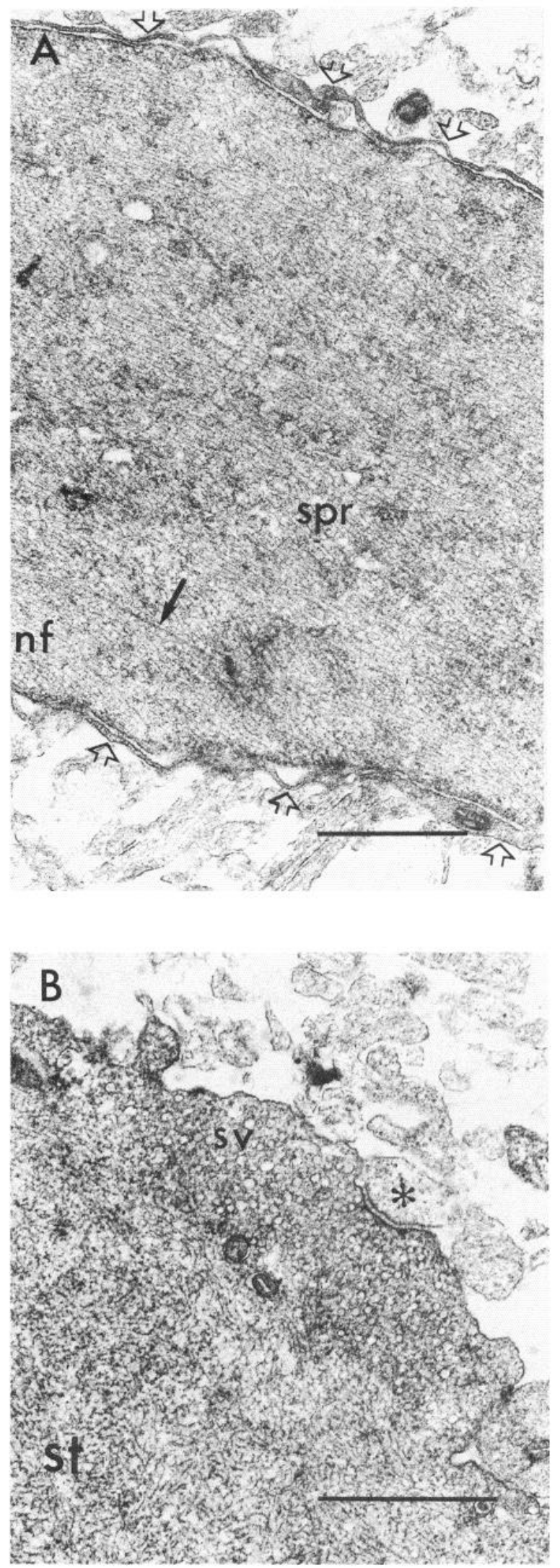

presynaptic element. The postsynaptic process (asterisk) is unidentified. Note that the neurofilaments in the sprout tip have a swirled appearance and appear disorganized compared with the longitudinally oriented neurofilaments in the sprout shaft shown in $A$ and in Figure 6. Scale bars, $1 \mu \mathrm{m}$. 

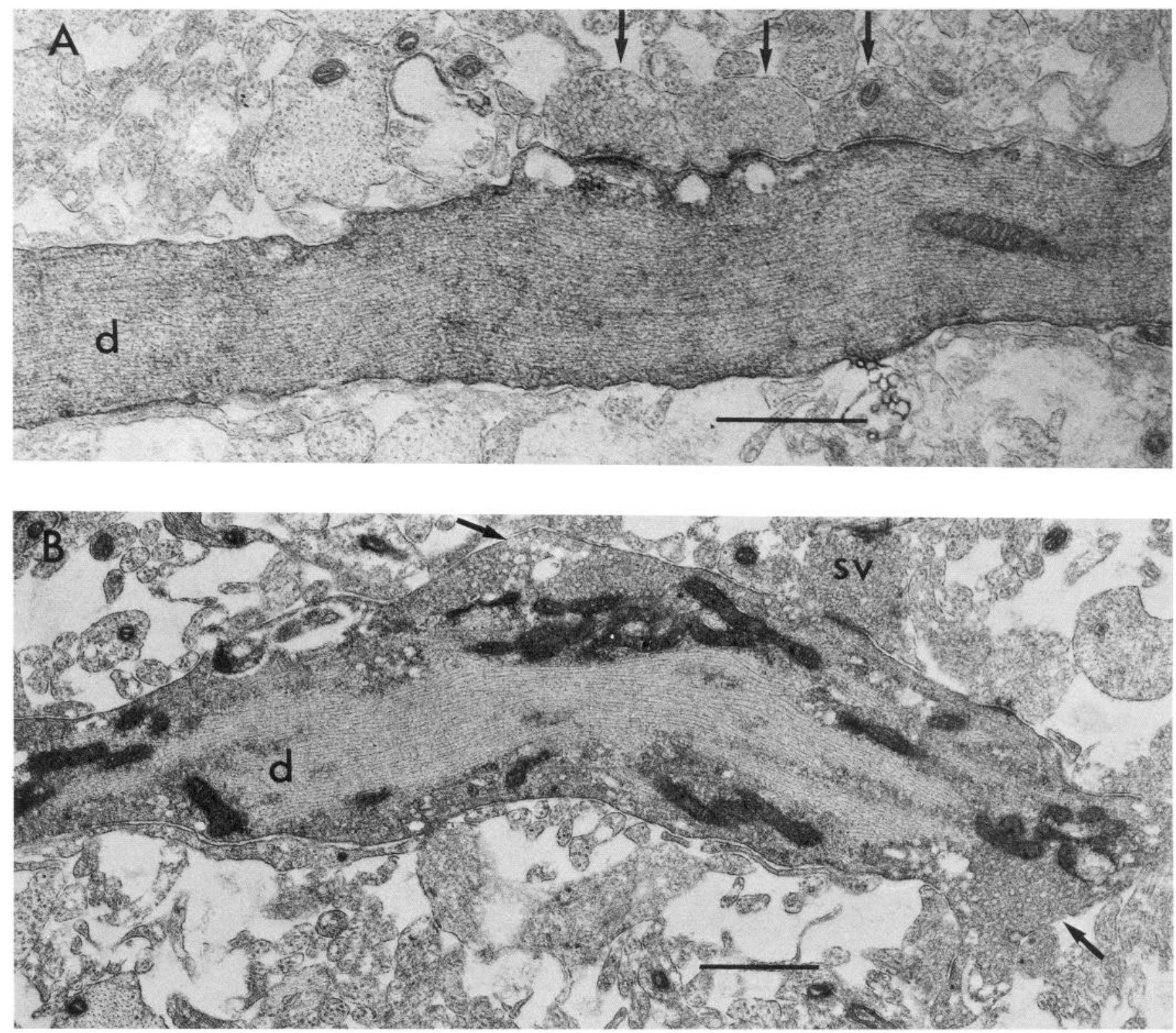

Figure 6. A, Longitudinal section of an intermediate ABC dendrite (d) $57 \mathrm{~d}$ after close axotomy. Note that the cytoskeleton consists almost entirely of neurofilaments aligned along the long axis of the dendrite, while microtubules are rare (compare with the dendrite of an intact ABC shown in Fig. 2). Dendrites of axotomized cells remain entirely postsynaptic to other neurons; here, synaptic boutons can be seen making contact with the HRP-filled dendrite. Scale bar, $1 \mu \mathrm{m}$. B, Longitudinal section of a distal dendrite from an ABC $70 \mathrm{~d}$ after close axotomy. Note the predominance of neurofilaments and the presence of a presynaptic terminal filled with synaptic vesicle $(s v)$ making synaptic contact with the dendrite. Large clusters of small vesicles not normally seen in dendrites (arrows) are also present. Scale bar, $1 \mu \mathrm{m}$.

Hirokawa et al. (1984) have shown that neurofilaments in neonatal axons and dendrites in the rabbit have lower levels of cross-bridging and tend to be more closely packed than are neurofilaments in mature axons. These differences were correlated with the presence of different ratios of the 3 major neurofilament proteins, with the protein associated with the crosslinks between neurofilaments being most prominent in mature axons. Such findings suggest that axotomy of lamprey ABCs may result in similar differences in neurofilament composition between newly generated axonal cytoskeleton (in sprouts and in dendrites of axotomized cells) and mature axonal cytoskeleton (in intact axons).

The sheath-like cellular elements associated with dendritic and axonal sprouts resemble the endfeet of radial glia in both the injured lamprey spinal cord (Wood and Cohen, 1981), and in the developing spinal tracts in Xenopus (Nordlander and Singer, 1982). This is interesting in view of the role that has been suggested for glial endfeet in the guidance of axons along the outer margin of the brain during embryogenesis in a number of systems (Singer et al., 1979; Krayanek and Goldberg, 1981; Nordlander and Singer, 1982; Silver and Rutishauser, 1984). It is possible that the glial elements which wrap neuritic sprouts in the lamprey provide the sprouts with "axonal" guidance cues that determine their linear, rostrocaudally oriented trajectories as suggested by Cohen and Hall (1986). However, it is not clear whether glial wrapping is the result of a specific recognition of 
"axonlike" sprouts by glial cells possessing the appropriate cues or a relatively nonspecific response to sprouting evoked by injury.

\section{Synapses made by dendritic sprouts}

The formation of synapses by axonlike sprouts originating from $\mathrm{ABC}$ dendrites raises the question of what role synapses may play in controlling sprout growth. These synapses occur in parts of the CNS where $\mathrm{ABC}$ axons are normally never found, so it is unlikely that dendritic sprouts are forming synapses on targets that $\mathrm{ABC}$ axons would ordinarily recognize in the course of normal embryogenesis. Growing neurites in the mollusc Helisoma have been reported to make transient synaptic contacts with inappropriate partners but make stable connections only with their contralateral homologs (Bulloch and Kater, 1982). The restriction of synapses to dendritic sprout tips is worthy of note in this context, as it implies that these synapses are transient structures that are progressively lost along the sprout shaft as the sprout tip advances. We have previously shown that dendritic sprouts evoked by close axotomy of ABCs reach a maximum length by $100 \mathrm{~d}$ postaxotomy and subsequently retract (Hall and Cohen, 1988a). In light of these data, it is tempting to speculate that dendritic sprouts evoked by axotomy of ABCs are only able to form transient synapses on targets that are different from their normal postsynaptic partners and that their inability to form stable synapses in foreign parts of the brain may eventually lead to sprout retraction.

\section{Ultrastructure of dendritic sprout tips}

The tips of dendritic sprouts resemble the axonal growth cones of some developing neurons both in their gross morphology (Nordlander and Singer, 1982; Tosney and Landmesser, 1985) and in some features of their ultrastructure, i.e., they contain many membranous vesicles (Del Cerro and Snider, 1968; Nutall and Wessells, 1979; Landis, 1983) and are often associated with glial clements (Nordlander and Singer, 1982; Silver and Rutishauser, 1984). However, the dendritic sprout tips of $\mathrm{ABCs}$ are remarkable in that they are filled with densely packed neurofilaments, which are not generally prominent in growth cones (reviewed by Landis, 1983). The absence of neurofilaments from regions of the cell that have high $\mathrm{Ca}^{2+}$ conductances (such as growth cones and presynaptic axonal terminals) has been attributed to the action of a calcium-dependent protease that breaks down neurofilaments (Schlaepfer and Hasler, 1979; Pant et al., 1982). This suggests that there may be a dynamic equilibrium between the orthograde transport of the polymerized filaments and their degradation as they reach the end of the neurite. Application of the protease inhibitor leupeptin has been shown to prevent the degradation of neurofilaments in the vicinity of axonal terminals, causing neurofilaments to accumulate there (Roots, 1983). It is possible that a similar shift in the equilibrium between neurofilament production and degradation is occurring in the dendritic sprouts described here, producing the accumulations of neurofilaments at the sprout tips.

Another question that should be addressed is whether the sprout tips we observed in the EM were actively growing at the time of fixation. Dendritic sprouts resulting from close axotomy of ABCs eventually retract; however, this usually becomes obvious only by $80 \mathrm{~d}$ or more following axotomy. It is possible that some of the sprout tips that we examined were in the early stages of retraction, which might be relevant to their anomalous ultrastructure. This is particularly true of the swollen sprout tips we examined, for swollen dendritic sprout tips become significantly more prevalent following the onset of sprout retraction (Hall and Cohen, 1988a). However, it is unlikely that all of the sprouts we observed would have been retracting by 57-70 d postaxotomy, and we found large numbers of neurofilaments in all of the sprout tips that we examined.

\section{Effect of close axotomy on dendritic ultrastructure}

A striking result of close axotomy described in this study was the virtually complete replacement of the dendritic cytoskeleton of ABCs with cytoskeletal elements typical of axons. This radical ultrastructural change occurred in the absence of major changes in gross dendritic morphology and was not accompanicd by the development in the dendrites of other features characteristic of axons or sprouts, such as presynaptic profiles or glial wrapping. The most straightforward explanation of this phenomenon is that elements of axonal cytoskeleton (consisting mainly of neurofilaments) are transported through the dendrites and into dendritic sprouts following close axotomy, replacing the preexisting microtubular cytoskeleton of the dendrites. Alternatively, the change might be due to conditions induced by close axotomy (such as changes in intracellular $\mathrm{Ca}^{2+}$ levels) that favor the depolymerization of microtubules and the polymerization of neurofilaments in the absence of major redistribution of cytoskeletal proteins. However, there is considerable evidence that neurofilament proteins are normally polymerized in both intact and regenerating neurons (reviewed by Lasek et al., 1983), suggesting that a large pool of unpolymerized neurofilament proteins is unlikely to be present in the dendrites under normal circumstances. Furthermore, the presence of large numbers of neurofilaments in dendritic sprouts suggests that considerable transportation of neurofilament proteins through the dendrites must occur following close axotomy (assuming that translation of neurofilament proteins occurs mainly in the cell body - see Lasek and Brady, 1981).

Polymerized neurofilaments appear to be transported as a bolus at the rate of slow axonal transport during axonal regeneration (Lasek et al., 1983; Hoffman et al., 1985), raising the question of whether a matrix of neurofilaments moves through $\mathrm{ABC}$ dendrites in a similar fashion following close axotomy. If so, one would expect to see a progressive changeover from microtubule- to neurofilament-dominated cytoskeleton both with time and as one moves from proximal to distal dendrites. In this study, we found no obvious differences between the cytoskeletal elements present in proximal and distal dendrites following close axotomy; all dendrites that we examined contained mostly neurofilaments. However, all of the ABCs we examined had been axotomized about 2 months previously and already exhibited extensive dendritic sprouting. We suspect that a complete changeover from the microtubular to neurofilamentous cytoskeleton may already have occurred by the time profuse dendritic sprouting begins, especially if polymcrizcd ncurofilaments must be transported en masse through the dendrites before they can be inserted into growing sprouts.

Another question raised by the results of this study is the fate of the original (predominately microtubular) dendritic cytoskeleton following close axotomy. The complete changeover from normal dendritic cytoskeleton to one with axonlike characteristics, combined with the lack of obvious changes in gross dendritic morphology in ABCs during the first 2 months after close axotomy (Hall and Cohen, 1988a), raises the possibility that the appearence of "new" cytoskeleton may be causally related to 
the disappearence of the "old" cytoskeleton. LeTerrier et al. (1982) have shown that the presence of neurofilament proteins specifically inhibits the polymerization of microtubules in vitro, apparently by binding to microtubule-associated proteins (MAPs) normally required for microtubule initiation and elongation. It is thus possible that the presence of axonal neurofilament proteins in the dendrites of axotomized ABCs might have a direct destabilizing effect on dendritic microtubules.

Although the exclusively postsynaptic status of the dendrites was unchanged by close axotomy, the number of incoming synapses was significantly reduced on the dendrites of axotomized cells. It is likely that some of this is due to partial deafferentation of ABCs by the lesion used to cause close axotomy. ABCs normally have strong synaptic inputs from the spinal cord (Rovainen, 1974; Wickelgren, 1977), some of which are very likely cut by lesions that hemisect the brain within $500 \mu \mathrm{m}$ of the $\mathrm{ABC}$ somata. On the other hand, the reduction of the number of synaptic inputs onto $\mathrm{ABCs}$ may also be due to effects of axotomy that reduce the ability of $\mathrm{ABC}$ dendrites to maintain their presynaptic connections. This is suggested by the observation that axotomy of motoneurons in the absence of direct injury to the CNS can also cause synapse "stripping" from the dendrites of affected cells (Kerns and Hinsman, 1973). The presence of a cytoskeleton-mediated, dendrite-specific intracellular transport system has recently been demonstrated in cultured hippocampal neurons (Davis et al., 1987). This invites one to speculate that close axotomy, by destroying most of the dendritic microtubules, impairs the ability of the dendrites to transport the materials needed to maintain specifically dendritic features such as postsynaptic relationships with other neurons.

\section{References}

Bartlett, W. P., and G. A. Banker (1984) An electron microscopic study of the development of axons and dendrites by hippocampal neurons in culture. 1. Cells which develop without intercellular contacts. J. Neurosci. 4: 1944-1953.

Batueva, I. V., and A. I. Shapovalov (1974) Synaptic effects evoked by supraspinal and intraspinal stimulation in lamprey motoneurons. Neurophysiology 6: 500-505.

Bray, D. (1973) Model for membrane movements in the neural growth cone. Nature 244: 93-96.

Bulloch, A. G. M., and S. B. Kater (1982) Neurite outgrowth and selection of novel electrical connections by adult Helisoma neurons. J. Neurophysiol. 48: 569-583.

Bunge, M. B. (1973) Fine structure of nerve fibers and growth cones of isolated sympathetic neurons in culture. J. Cell Biol. 56: 713-735.

Chamak, B., A. Fellous, J. Glowinski, and A. Prochiantz (1987) MAP-2 expression and neuritic outgrowth and branching are coregulated through region-specific neuro-astroglial interactions. J. Neurosci. 7: 3163-3171.

Chang, T. P. O., and T. S. Reese (1986) Polarized compartmentalization of organelles in growth cones from developing optic tectum. J. Cell Biol. 85: 1473-1480.

Christensen, B. N. (1976) Morphological correlates of synaptic transmission in lamprey spinal cord. J. Neurophysiol. 39: 197-212.

Ciment, G., A. H. Ressler, P. C. Letourneau, and J. A. Weston (1986) A novel neurofilament associated protein, NAPA-73. J. Cell Biol. 102: 246-251.

Cohen, M. J., and G. F. Hall (1986) Control of neuron shape during development and regeneration. Neurochem. Pathol. 5: 331-343.

Davis, L., G. A. Banker, and O. Steward (1987) Selective dendritic transport of RNA in hippocampal neurons in culture. Nature 330: 477-479.

Del Cerro, M. P., and R. S. Snider (1968) Studies of the developing cerebellum. Ultrastructure of the growth cones. J. Comp. Neurol. 133: 341-362.

Dotti, C. G., and G. A. Banker (1987) Experimentally induced alteration in the polarity of developing neurons. Nature 330: 254-256.
Fishman, P. S. (1975) A study of dendritic form in identified lamprey neurons. Ph.D. dissertation, Yale University, New Haven.

Grafstein, B., and I. G. MacQuarrie (1978) The role of the nerve cell body in axonal regeneration. In Neuronal Plasticity, C. Cotman, ed., pp. 155-195, Raven, New York.

Hall, G. F., and M. J. Cohen (1983) Extensive dendritic sprouting induced by close axotomy of central neurons in the lamprey. Science 222: 518-521.

Hall, G. F., and M. J. Cohen (1988a) The pattern of dendritic sprouting and retraction induced by axotomy of lamprey central neurons. $J$. Neurosci. 8: 3584-3597.

Hall, G. F., and M. J. Cohen (1988b) Dendritic amputation redistributes sprouting evoked by axotomy in lamprey central neurons. J. Neurosci. 8: 3598-3606.

Hirokawa, N., M. A. Glicksman, and M. B. Willard (1984) Organization of mammalian neurofilament peptides within the neuronal cytoskeleton. J. Cell Biol. 98: 1523-1536.

Hoffman, P. N., G. W. Thompson, J. W. Griffin, and D. L. Price (1985) Changes in neurofilament transport coincide temporally with alterations in the caliber of axons in regenerating motor fibers. J. Cell Biol. 101: $1332-1340$.

Kerns, J. M., and E. J. Hinsman (1973) Neuroglial response to sciatic neurectomy 2: Electron microscopy. J. Comp. Neurol. 151: 255-280.

Krayanek, S., and S. Goldberg (1981) Oriented extracellular channels and axonal guidance in the embryonic chick retina. Dev. Biol. 84 41-50.

Landis, S. C. (1983) Neuronal growth cones. Annu. Rev. Physiol. 45: $567-580$.

Lasek, R. J., and S. T. Brady (1981) The axon: A prototype for studying expressional cytoplasm. Cold Spring Harbor Symp. Quant. Biol. 46: 113-124.

Lasek, R. J., M. M. Oblinger, and P. F. Drake (1983) Molecular biology of neuronal geometry: Expression of neurofilament genes influences axonal diameter. Cold Spring Harbor Symp. Quant. Biol. 48: 731744.

LeTerrier, J. F., R. K. H. Leim, and M. Shelanski (1982) Interactions between neurofilament and microtubulc associated proteins: A possible mechanism for intraorganelle bridging. J. Cell Biol. 95: 982986.

Linda, H., M. Risling, and S. Cullheim (1985) "Dendraxons" in regenerating motoneurons in the cat: Do dendrites generate new axons after central axotomy? Brain Res. 358: 329-333.

Llinás, R. R. (1979) The role of calcium in neuronal function. In The Neurosciences: Fourth Study Program, F. O. Schmitt and F. G. Worden, eds., pp. 555-571, MIT Press, Cambridge, MA.

MacVicar, B. A., and R. R. Llinás (1985) Barium action potentials in regenerating axons of the lamprey spinal cord. J. Neurosci. Res. 13: 323-335.

Martin, R. J. (1979) A study of the morphology of the large reticulospinal neurons of the lamprey ammocoete by intracellular injection of Procion Yellow. Brain Behav. Evol. 16: 1-18.

Matus, A., R. Bernhardt, and T. Hugh-Jones (1981) High molecular weight microtubule associated proteins are preferentially associated with dendritic microtubules in the brain. Proc. Natl. Acad. Sci. USA 78: 3010-3014.

Murphy, A. D., and S. B. Kater (1980) Sprouting and functional regeneration of an identified neuron in Helisoma. Brain Res. 186: 251272.

Nordlander, R. H., and M. Singer (1982) Morphology and position of growth cones in the developing Xenopus spinal cord. Dev. Brain Res. 4: 181-193.

Nutall, R. P., and N. K. Wessells (1979) Veils, mounds and vesicle aggregates in neurons elongating in vitro. Exp. Cell Res. 119: 163174.

Pant, H. C., P. E. Gallant, R. Gould, and H. Gaincr (1982) Distribution of calcium activated protease activity and endogenous substrates in the squid nervous system. J. Neurosci. 2: 1578-1587.

Peters, A., S. L. Palay, and H. D. Webster (1976) The Fine Structure of the Nervous System: The Neurons and Supporting Cells. W. B. Saunders, Philadelphia.

Pfenninger, K. H., and M. P. Johnson (1983) Membrane biogenesis in the sprouting neuron. 1: Selective transfer of newly synthesized phospholipid into the growing neurite. J. Cell Biol. 97: 1038-1042.

Pfenninger, K. H., and M.-F. Maylie-Pfenninger (1981) Lectin labelling of sprouting neurons. 2: Relative movement and appearance 
of glycoconjugates during plasmalemmal expansion. J. Cell Biol. 89: 547-559.

Roederer, E., and M. J. Cohen (1983) Regeneration of an identified central neuron in the cricket. I. Control of sprouting from soma, dendrites, and axon. J. Neurosci. 3: 1835-1847.

Roots, B. (1983) Neurofilament accumulations induced in synapses by leupeptin. Science 221: 971-972.

Rovainen, C. M. (1974) Synaptic interactions of reticulospinal neurons and nerve cells in the spinal cord of the sea lamprey. J. Comp. Neurol. 154: 207-224.

Schacher, S., and E. Proshansky (1983) Neurite regeneration by Aplysia neurons in dissociated cell culture: Modulation by Aplysia hemolymph and the presence of the initial axon segment. J. Neurosci. 3: 2403-2413.

Schlaepfer, W. W., and M. B. Hasler (1979) Characterization of the calcium induced disruption of neurofilaments in rat peripheral nerve. Brain Res. 168: 299-309.

Siegel, S. (1956) Nonparametric Statistics for the Behavioral Sciences, pp. 152-158, McGraw-Hill, New York.

Silver, J., and U. Rutishauser (1984) Guidance of optic axons in vivo by a preformed adhesive pathway on neuroepithelial endfeet. Dev. Biol. 106: 485-499.
Singer, M., R. H. Nordlander, and M. Egar (1979) Axonal guidance during embryogenesis and regeneration in the spinal cord of the newt. The blueprint hypothesis of neuronal pathway patterning. J. Comp. Neurol. 185: 1-22.

Skoff, R. P., and V. Hamburger (1974) Fine structure of dendritic and axonal growth cones in embryonic chick spinal cord. J. Comp. Neurol. 153: 107-148.

Stewart, W. H. (1978) Functional connections between cells as revealed by dye-coupling with a highly fluorescent napthalimide. Cell 14: 741-759.

Tosney, K. W., and L. T. Landmesser (1985) Growth cone morphology and trajectory in the lumbosacral region of the chick embryo. J. Neurosci. 5: 2345-2358.

Wickelgren, W. O. (1977) Physiological and anatomical characteristics of reticulospinal neurones in lamprey. J. Physiol. (Lond.) 270: 89116.

Wood, M. R., and M. J. Cohen (1979) Synaptic regeneration in identified neurons of the lamprey spinal cord. Science $206: 344-347$.

Wood, M. R., and M. J. Cohen (1981) Synaptic regeneration and glial reactions in the transected spinal cord of the lamprey. J. Neurocytol. $10: 57-79$. 